\title{
Rettungsdienst, der Libero - Armut und soziale Not
}

Gerhard Trabert

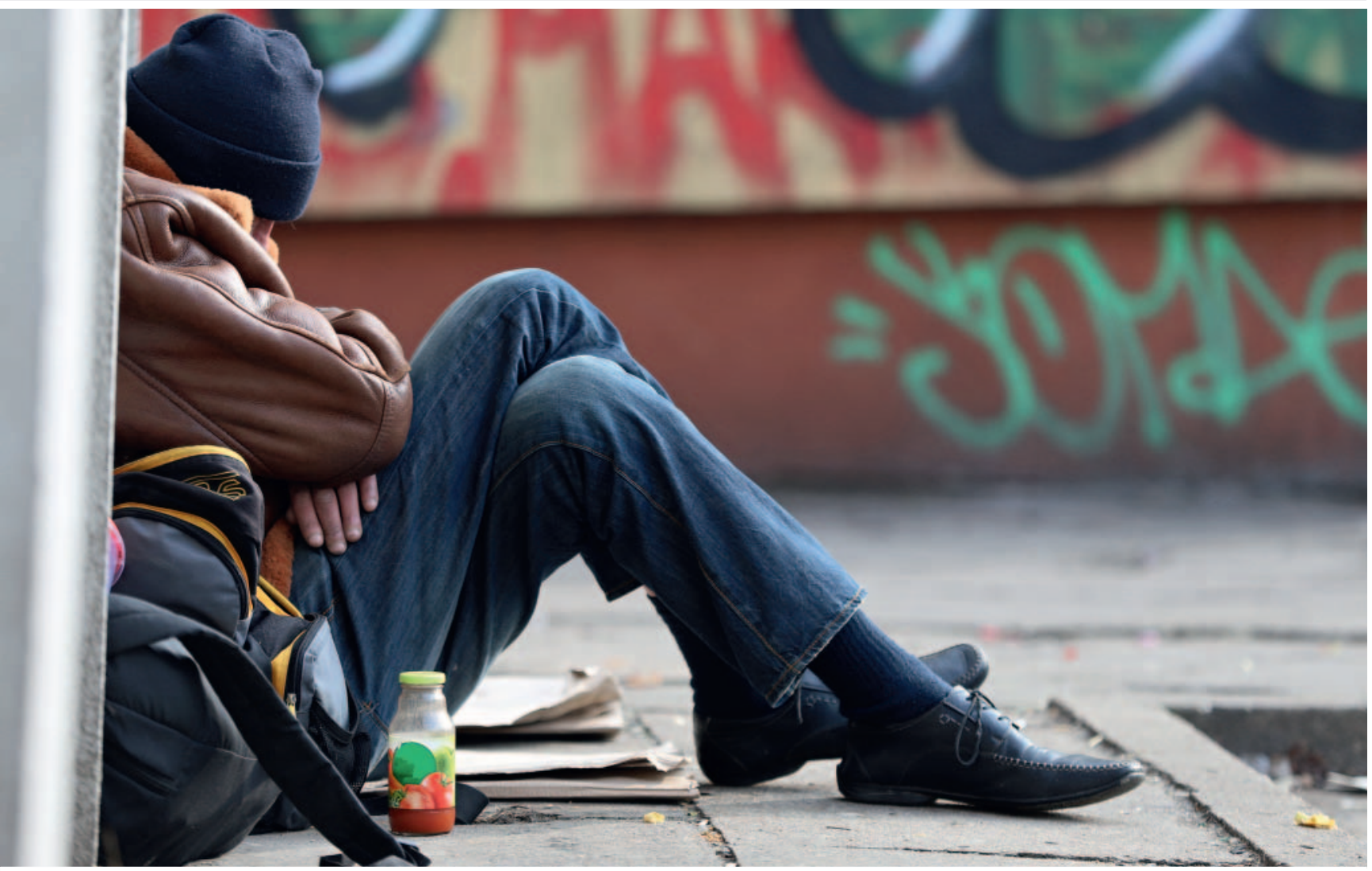

Quelle: Robert/adobe.stock.com (Symbolbild)

In der Begegnung mit Menschen, die von Armut und Ausgrenzung betroffen sind, kann der Rettungsdienst viel richtig und viel falsch machen. Dieser Artikel versucht einige Hinweise und Handlungsempfehlungen zu vermitteln und zudem das Bewusstsein sowie die Sensibilität zum Thema Armut und deren Korrelation zu Krankheit zu verstärken.

„Armut ist die schlimmste Form von Gewalt.“ (Mahatma Gandhi)

Armut nimmt auch in Deutschland stetig zu. Der Rettungsdienst im Gesundheitswesen wird immer häufiger zu Menschen gerufen, die sich in einer sozialen Notlage befinden und häufig infolgedessen auch körperlich und seelisch erkrankt sind bzw. höhere Prävalenzen für Erkrankungen haben. Das überfordert den Rettungsdienst oft in seiner Kompetenz; nicht immer kann er all die Hilfe leisten, die notwendig wäre. Denn im Mittelpunkt der Notwendigkeit steht nicht immer die gesundheitliche Intervention, sondern v. a. ein Bedürfnis nach psychoso- zialer Unterstützung. Demzufolge wird ein solcher Hilfseinsatz (zu) oft (zu) schnell als Fehleinsatz deklariert.

Merke

Auch wenn der Rettungsdienst fehlende psychosoziale Versorgungsstrukturen aufdeckt und mehr oder weniger gut versucht zu kompensieren, kann er in der Begegnung mit den von Armut und Ausgrenzung betroffenen Menschen viel richtig und viel falsch machen. 


\section{Wer ist arm?}

Es existiert keine eindeutige Armutsdefinition. Generell wird zwischen absoluter Armut (die physische Existenz bedrohend) und relativer Armut unterschieden. Definitionsversuche relativer Armut in Deutschland orientieren sich schwerpunktmäßig an der finanziellen Ausstattung. Daher wird von Einkommensarmut gesprochen.

Laut europäischer Definition sind Menschen von strenger Armut betroffen, wenn sie $40 \%$ oder weniger des durchschnittlichen monatlichen Haushaltseinkommens besitzen. Dazu gehört jeder Bezieher von sozialen Transferleistungen, also von Arbeitslosengeld II oder Sozialgeld. Davon betroffen sind in Deutschland insbesondere Kinder und Jugendliche, Alleinerziehende (i. d. R. Mütter), Familien mit mehr als 3 Kindern, arbeitslose Menschen sowie ausländische Mitbürger.

\section{Armut und Krankheit}

Konkrete Zusammenhänge zwischen dem sozialen Status und Krankheit konnten u. a. für das Auftreten von koronaren Herzkrankheiten (Herzinfarkt: 2- bis 3-fach erhöhtes Risiko), Schlaganfall (ebenfalls 2- bis 3-fach erhöhtes Risiko), Krebserkrankungen und Lebererkrankungen festgestellt werden. Erkrankungen der Verdauungsorgane (Magengeschwüre) und der Atmungsorgane (Lungenentzündungen, chronische Bronchitis) findet man ebenfalls häufiger als im Bevölkerungsdurchschnitt. Des Weiteren ist die Infektanfälligkeit erhöht. Zusätzlich treten psychiatrische Erkrankungen in den Vordergrund, darunter besonders Depressionen bis zum Suizid [1-7].

Neben der Morbidität ist auch die Mortalität von Armut betroffener Menschen in unserer Gesellschaft erhöht. So besteht ein Lebenserwartungsunterschied von 11 Jahren bei den Männern und 8 Jahren bei den Frauen zwischen dem reichsten und dem ärmsten Viertel der deutschen Bevölkerung (Deutsches Institut für Wirtschaftsforschung; Erhebung im 5-Jahreszeitraum; siehe auch [4, 8]). Erschwerend kommt hinzu, dass es immer noch eine Unkultur der Diffamierung und Schuldzuweisung gegenüber sozial Benachteiligten gibt, die häufig dazu führt, dass die Betroffenen ihren Selbstwert infrage stellen.

\section{Inanspruchnahme des Notarztdienstes}

Eine der ersten Studien zum Inanspruchnahmeprofil des Notarztes mit einem besonderen Augenmerk auf die soziale Schichtzugehörigkeit wurde 2002 von Luiz et al. [9] in Kaiserslautern durchgeführt. Die einzelnen Stadtbezirke wurden nach der sozioökonomischen Bewohnerstruktur differenziert. In den als schlecht situierte Wohnbezirke charakterisierten Regionen kam es zu einer signifikanten Häufung von psychosozialen und auch psychiatrischen Ein- sätzen. Außerdem unterschied sich das Altersprofil: Während im Gesamtdurchschnitt v. a. Personen höheren Alters den Notarzt rufen, waren es bei den Einsätzen mit einer psychosozialen Indikation Personen mittleren Erwachsenenalters. Jeder 8. Notarzteinsatz war der Beschreibung „psychosozialer Notfall“ zuzuordnen.

Eine Datenanalyse aus Berlin [10] bestätigt, dass in Stadtteilen mit einem hohen Anteil sozial benachteiligter Menschen, die z. B. von ökonomischer Armut und Arbeitslosigkeit betroffen sind, Notfalleinsätze signifikant häufiger stattfinden. Luiz beschreibt diese Realität im Rettungswesen wie folgt: „... als niederschwellig erreichbare, unmittelbar und flächendeckend verfügbare ärztliche Institution primär mit nichtlebensbedrohlichen Notfällen und Krisensituationen der unterschiedlichen Art konfrontiert. (...) So wird der Einsatzalltag vielerorts immer häufiger von den psychischen und somatischen Konsequenzen eines Versagens sozialer Ressourcen geprägt“ [9]. Er stellt dabei aber auch fest: „Der Vorwurf, mit dem Einsatz des Notarztes bei psychosozialen Notfällen eine ,hochwertige Ressource‘ zu vergeuden, ist (...) solange ungerechtfertigt, wie die Notfallmedizin das Fehlen komplementärer sozialer und medizinischer Einrichtungen ausgleichen muss“ [9].

\section{Menschliche Zuwendung}

Zur Beziehung zwischen der Inanspruchnahme von Notfallambulanzen und sozialen Notsituationen hat eine kanadische Studie interessante Erkenntnisse beschrieben. Hintergrund der epidemiologischen Untersuchung war die Annahme vieler Ärzte in der Notfallaufnahme, durch zu große Freundlichkeit obdachlosen Menschen gegenüber könnten sie ein häufigeres Aufsuchen der Notfallambulanz ohne ernsthafte Beschwerden mitverursachen. Daraufhin wurde untersucht, wie sich eine einfühlsame, betroffenenzentrierte Behandlung wohnungsloser Menschen, die aufgrund akuter Beschwerden die Notfallambulanz eines Krankenhauses in Toronto aufsuchten, auf die Häufigkeit weiterer Konsultationen auswirkt.

133 wohnungslose Männer wurden in 2 Gruppen aufgeteilt: Die eine Gruppe wurde vom Krankenhauspersonal in der üblichen Weise betreut. Die andere Gruppe wurde zusätzlich geschulten Freiwilligen zugeteilt, die sich während der Wartezeit mit ihnen über ihre Beschwerden unterhielten, Anteil nahmen und Empathie zeigten und die wohnungslosen Patienten zu einem Getränk und einer kleinen Mahlzeit einluden. Das für viele erstaunliche Ergebnis: Einfühlsam betreute wohnungslose Männer suchten in der Folgezeit die Notfallambulanz signifikant seltener auf als die Patienten der Kontrollgruppe, die in der üblichen Weise behandelt worden waren. 
Eine der Erklärungen der Forschungsgruppe: Patienten und speziell von Armut betroffene Patienten gehen häufiger zum Arzt, wenn sie mit dem Ergebnis des Arztkontakts nicht zufrieden waren. Empathische Anteilnahme ist gerade für sozial benachteiligte Patienten eine Reaktionsweise, die sie sich von Medizinern erhoffen. Die gewünschte Erfahrung führt dann zu einer selteneren Kontaktsuche [11].

Merke

Einfühlsame Behandlung sozial benachteiligter Menschen in der Notfallambulanz und im Rettungsdienst ist demnach nicht nur menschlich, sondern auch ökonomisch sinnvoll.

\section{Arm sein ist ein Trauma}

Die tertiäre Traumatisierung oder auch das Modell der sequenziellen Traumatisierung nach Hans Keilson [12] bezieht sich auf den Umgang mit Traumatisierten und von Armut Betroffenen. Sie sind in einer der reichsten Gesellschaften oft traumatisiert. Die Art der Begegnung zwischen Helfer und Traumatisiertem kann dabei für die Verarbeitung eines Traumas ausschlaggebender sein als das traumatische Ereignis selbst: Die eventuell stattfindende sog. tertiäre Traumatisierung ist entscheidend für die Ausbildung einer Traumatisierungsreaktion bzw. Chronifizierung psychischer und physischer Beeinträchtigungen.

Entscheidend in dieser „dritten Phase“ der Trauma-Arbeit ist, dass soziale Sicherheit und Stabilität erfahren wird. Das umfasst ein empathisches, authentisches, von Ernsthaftigkeit geprägtes Beziehungskonzept. Dies wiederum ist eine interdisziplinäre Aufgabe, in der auch der Rettungsdienst eine wichtige Rolle spielen kann. Der Patient muss sich wertgeschätzt und ernst genommen fühlen. Somit ist der Rettungsdienst eine erste wichtige Kontaktstelle, die eine weitere Traumatisierung bahnen oder verhindern kann.

\section{Akutmedizin - ganz praktisch}

Als Notarzt und Sozialarbeiter möchte ich im folgenden Abschnitt einige konkrete praktische Hinweise für den akutmedizinischen Umgang mit sozial benachteiligten Patienten bzw. Menschen in sozialen Notlagen auflisten:

- Die Lebenslage der Patienten mitberücksichtigen (Ganzheitsmedizin)!

- Kann der Patient schriftliche Anweisungen/Informationen lesen? (Zunehmende Zahl von Analphabeten bzw. funktionellen Analphabeten. Funktionelle Analphabeten können lesen und schreiben, verstehen aber sehr häufig die inhaltliche Aussage des Gelesenen nicht.)

- Genaue nachfragende Anamnese, leicht verständliche und nachvollziehbare Sprache. (Konzept der „leichten Sprache“)
- Gesundheitsrisikoverhalten und die damit einhergehenden Gefahren berücksichtigen: Zigaretten- und Alkoholkonsum. An äthyltoxische Polyneuropathien und Vitamin-B12- sowie Folsäure-Mangel denken.

- Ernährungsgewohnheiten erfragen.

- Impfstatus oft lückenhaft! Nachfragen und Impflücken schließen. (Im Rettungsdienst ist die Tetanusimpfung von besonderer Bedeutung.)

- Aufgrund einer oft unzureichenden Nahrungszufuhr und Alkoholproblematik besteht eine erhöhte Hypoglykämie-Gefahr! Deshalb immer Blutzucker- und Blutdruckmessung.

- Auf der Straße bzw. in Armut zu leben bedeutet Stress. Deshalb an typische Stresserkrankungen denken: z. B. Hypertonie, Koronarsyndrom, Ulcera pepticum (Magengeschwüre). (Die Frage nach vorhandenem Teerstuhl als diagnostischem Hinweis auf eine obere gastrointestinale Blutung ist fast obligatorisch.)

- Multiple Gefahrenquelle Alkohol: Z. B. wird Alkohol im Winter als Wärmespender genutzt, des Weiteren als Nahrungsersatz und zur Schmerzunterdrückung.

- Gefahr der Fehlinterpretation von Beschwerden aufgrund der Tendenz, bei Menschen in sozialen Notlagen Symptome als Ausdruck einer Alkoholkrankheit zu interpretieren und damit Akuterkrankungen zu übersehen (z. B. Sturz mit zerebraler Blutung).

- Patienten immer in entkleidetem Zustand untersuchen, auch wenn dies manchmal mit unangenehmen Gerüchen verbunden sein kann (u. a. hohe Prävalenz dermatologischer Erkrankungen, parasitäre Erkrankungen, Autoaggressivität usw.).

- Parasitäre Erkrankungen: Neben der Behandlung des Patienten auch das Umfeld, Freunde, Bekannte, Familienmitglieder, Betreuungspersonal (z. B. Sozialarbeiter) behandeln bzw. zumindest informieren. Kleider unter Umständen entsorgen. An Skabies und Pediculus (Kopfläuse) denken!

- An psychiatrische Erkrankungen und Auffälligkeiten denken! Psychotische paranoide Erkrankungsbilder, Depressionen mit einer erhöhten Suizidgefahr, autoaggressives Verhalten (Selbstverletzung, BorderlinePatienten).

- An Selbstgefährdung (s. o.) und Fremdgefährdung denken (u. a. Gewaltbereitschaft nach erhöhtem Konsum legaler sowie illegaler Drogen).

- Jeder Akutpatient, der sich in einer sozialen Notlage befindet, gehört im Krankenhaus intensiv klinisch und labordiagnostisch, ggf. auch funktionsdiagnostisch untersucht!

- Zahnerkrankungen sind sehr häufig: Deshalb den Zahnstatus überprüfen und eine entsprechende Sanierung ansprechen und initiieren. Pathologische Zahnbefunde als chronische bakterielle Streuherde berücksichtigen. 
Cave

Medikamente mit suchterzeugenden Stoffen, Hustensäfte mit Alkoholanteilen! Etwaige Wechselwirkungen zwischen Alkohol und Medikamenten beachten (u. a. Neuroleptika, Antiepileptika, Antihistaminika, Sedativa usw.).

\section{Weiterbetreuung und Versorgung}

Die Vernetzung mit alternativen medizinischen sowie psychosozialen Institutionen bzw. Versorgungsstrukturen ist für den Rettungsdienst eine wichtige Aufgabe. Sie kann einerseits Entlastung schaffen, andererseits eine adäquatere und somit kompetentere Versorgung bedeuten. Problematisch ist, dass solche gesundheitlichen bzw. psychosozialen Versorgungsangebote oft vor Ort nicht sofort hinzugezogen werden können. Dann müssen ganz neue Kooperations- und Umsetzungsmöglichkeiten und Wege entwickelt werden.

\section{PRAXIS/MABNAHMEN}

Listen von psychosozialen und karitativen Einrichtungen anlegen und im Rettungsdienst nutzen!

\section{FAZIT}

Gesundheitsversorgung, auch die notfallmäßige Versorgung, ist ein Menschenrecht, gerade auch für sozial benachteiligte Menschen! Dabei kann man (auch im Rettungsdienst) viel falsch, aber auch sehr viel richtig machen. Eine unvoreingenommene Haltung und praktisches Wissen helfen dabei, sozial benachteiligte Menschen gut und effektiv zu versorgen.

\section{Interessenkonflikt}

Die Autoren geben an, dass kein Interessenkonflikt besteht.

\section{Autorinnen/Autoren}

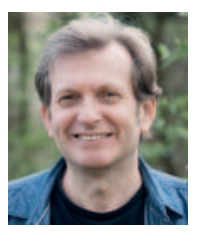

\section{Gerhard Trabert}

Prof. Dr. med. Dipl.-Sozialpädagoge. Arzt für Allgemeinmedizin/Notfallmedizin, Professor für Sozialmedizin/Sozialpsychiatrie, Sozialpädagoge, Autor. Gründer des Vereins Armut und Gesundheit in Deutschland. Initiator der Ambulanz ohne Grenzen in Mainz. Leiter

der Arbeitsgruppe Armut und Gesundheit der Nationalen Armutskonferenz Deutschlands.
Korrespondenzadresse

Prof. Dr. Gerhard Trabert

Armut und Gesundheit

Zitadelle Bau $1 \mathrm{~F}$

55131 Mainz

gerhard.trabert@hs-rm.de

Literatur

[1] Mielck A. Soziale Ungleichheit und Gesundheit. Empirische Ergebnisse, Erklärungsansätze, Interventionsmöglichkeiten. Bern: Huber; 2000

[2] Mielck A. Soziale Ungleichheit und Gesundheit. In: Hurrelmann K, Kolip P, Hrsg. Geschlecht, Gesundheit und Krankheit. Männer und Frauen im Vergleich. Bern: Huber; 2000

[3] Mielck A. Soziale Ungleichheit und Gesundheit. Einführung in die aktuelle Diskussion. Bern: Huber; 2005

[4] Helmert U, Bammann K, Voges W et al. Hrsg. Müssen Arme früher sterben? Soziale Ungleichheit und Gesundheit in Deutschland. Weinheim: Beltz Juventa; 2000

[5] Mackenbach JP, Bakker MJ, Kunst AE et al. Socioeconomic inequalities in health in Europe - An overview. In: Mackenbach JP, Bakker MJ, eds.; Reducing inequalities in health: a European perspective. London: Routledge; 2002: 3-24

[6] Bauer U, Bittlingmayer UH, Richter M Hrsg. Health Inequalities - Determinanten und Mechanismen gesundheitlicher Ungleichheit. Wiesbaden: VS-Verlag; 2008

[7] Sachverständigenrat zur Begutachtung der Entwicklung im Gesundheitswesen Hrsg. Koordination und Qualität im Gesundheitswesen. Band 1: Kooperative Koordination und Wettbewerb, Sozioökonomischer Status und Gesundheit, Strategien der Primärprävention. Stuttgart: Kohlhammer; 2006

[8] Lampert T, Kroll L. Armut und Gesundheit. Zahlen und Trends aus der Gesundheitsberichterstattung des Bundes; Robert Koch-Institut Berlin; GBE kompakt 5/2010

[9] Luiz T, Schmitt TK, Madler C. Der Notarzt als Manager sozialer Krisen. Notf Rett Med 2002; 5: 505-511

[10] Poloczek S, Arntz B. Tagung „Medizin im Kontext“. 19. Mai 2007 Berlin

[11] Redelmeier DA, Molin JP, Tibshirani RJ. A randomised trial of compassionate care for the homelessl in an emergency department. Lancet 1995; 345: 1131-1134

[12] Keilson H. Sequentielle Traumatisierung bei Kindern. Untersuchungen zum Schicksal jüdischer Kriegswaisen. Gießen: Edition Psychosozial, Psychosozial Verlag; 2005

[13] Trabert G, Wagner U. Patienten in Sozialnot. Besondere Personengruppen im Rettungsdienst. Karutz H, Schröder S, Hrsg. Edewecht: Stumpf + Kossendey; 2017

Bibliografie

DOI https://doi.org/10.1055/a-0970-6218

retten 2020; 9: 6-9

(c) Georg Thieme Verlag KG, Stuttgart · New York ISSN 2193-2387 